\title{
Improved Quantum Hard-Sphere Ground-State Equations of State
}

\author{
M.A. Solís ${ }^{1,2}$, M. de Llano ${ }^{3}$, J.W. Clark ${ }^{1}$, and George A. Baker, Jr. ${ }^{4}$ \\ ${ }^{1}$ Department of Physics, Washington University \\ St. Louis, Missouri 63130, USA \\ ${ }^{2}$ Instituto de Física, Universidad Nacional Autónoma de México \\ Apdo. Postal 20-364, 01000 México, D.F., Mexico \\ ${ }^{3}$ Instituto de Investigaciones en Materiales, Universidad Nacional Autónoma de México, \\ Apdo. Postal 70-360, 04510 México, DF, Mexico \\ \& \\ Consortium of the Americas for Interdisciplinary Science, \\ University of New Mexico, Albuquerque, NM 87131, USA \\ ${ }^{4}$ Theoretical Division, University of California, \\ Los Alamos National Laboratory, Los Alamos, NM 87545, USA
}

November 19, 2018

\begin{abstract}
The London ground-state energy formula as a function of number density for a system of identical boson hard spheres, corrected for the reduced mass of a pair of particles in a "sphere-of-influence" picture, and generalized to fermion hardsphere systems with two and four intrinsic degrees of freedom, has a double-pole at the ultimate regular (or periodic, e.g., face-centered-cubic) close-packing density usually associated with a crystalline branch. Improved fluid branches are contructed based upon exact, field-theoretic perturbation-theory low-density expansions for many-boson and many-fermion systems, extrapolated to intermediate densities via Padé and other approximants, but whose ultimate density is irregular or random closest close-packing as suggested in studies of a classical system of hard spheres. Results show substantially improved agreement with the best available Green-function Monte Carlo and diffusion Monte Carlo simulations for bosons, as well as with ladder, variational Fermi hypernetted chain, and so-called L-expansion data for two-component fermions.
\end{abstract}

PACS: 67.40.Db; 64.10.+h; 05.30.Fk; 05.30.Jp; 05.70.Ce

Key words: boson and fermion hard-sphere fluids; random close-packing; liquid ${ }^{4} \mathrm{He}$ and ${ }^{3} \mathrm{He}$; nuclear and neutron matter.

\section{Introduction}

An analytical formula for the ground-state energy $E$ of an $N$-hard-sphere-boson system of volume $\Omega$ for all particle-number densities $\rho \equiv N / \Omega$ was proposed by London [1] as

$$
E / N=\frac{2 \pi \hbar^{2} c}{m} \frac{1}{\left(\rho^{-1 / 3}-\rho_{0}^{-1 / 3}\right)^{2}} \frac{1}{\left(\rho^{-1 / 3}+b \rho_{0}^{-1 / 3}\right)}
$$

where $m$ is the particle mass, $c$ is the hard-sphere diameter and the constant $b$ equals $2^{5 / 2} / \pi-1$. Here, $\rho_{0} \equiv \sqrt{2} / c^{3}$ is the assumed ultimate regular (or periodic) close-packing density at which a system of identical classical hard spheres close-pack in a primitive-hexagonal arrangement, e.g., face-centered-cubic or hexagonal. As remarked by Rogers 2 this is what "many mathematicians believe and all physicists know" to be the case. However, the Kepler 1611 conjecture [3] that $\rho_{0} \equiv \sqrt{2} / c^{3}$ is the ultimate packing density for identical hard spheres seems to be approaching theorem status [4] after many attempts of proof. 
The justification given for (1) is that it reduces smoothly to limiting expressions at both low and high densities, namely

$$
\begin{gathered}
E / N \underset{\rho \rightarrow 0}{\longrightarrow}\left(2 \pi \hbar^{2} / m\right) \rho c \\
E / N \underset{\rho \rightarrow \rho_{0}}{\longrightarrow} A\left(\hbar^{2} / 2 m\right)\left(\rho^{-1 / 3}-\rho_{0}^{-1 / 3}\right)^{-2}
\end{gathered}
$$

but gives no indication of a "freezing" or Kirkwood [5] phase transition at some number density $\rho$ between 0 and $\rho_{0}$. Here $A=\pi^{2} / 2^{1 / 3} \simeq 7.8335$ is a constant called the residue of the second-order (or double) pole at close packing. Using the polyhedron cell method suggested in Ref. [6], the value of $A$ has been predicted [7] theoretically to lie within the rigorous range

$$
1.63 \leq A \leq 27.0 .
$$

The low-density leading term (2) is the celebrated Lenz 8 term, calculated by him as the leading correction to the energy arising from an "excluded volume" effect. The Lenz term has finally been rigorously established [9]. The limit (3) comes from the lowest Schrödinger equation eigenvalue of a particle in a spherical cavity, and is just the kinetic energy of a point particle of mass $m$ inside the cavity of radius $r-c$, where $r$ is the average separation between two neighboring hard spheres and $r=(\sqrt{2} / \rho)^{1 / 3}$ by assuming a primitive-hexagonal packing arrangement for the cavities.

More recently it was found [10, however, that the arguments leading to the high-density limit of the original [1] (boson) London equation (11) are flawed by a fundamental error: the spherical cavity of radius $r-c$ alluded to above in reality refers to the "sphere of influence" of two particles. Thus, the particle mass used in obtaining (3) should refer to the reduced mass $m / 2$ of the pair. This yields the constant

$$
b \equiv 2^{3 / 2} / \pi-1
$$

instead of the constant $2^{5 / 2} / \pi-1$ given by London for (11). The result (1) with (15) is designated the modified London $(M L)$ equation. It continues to satisfy (2) as this is independent of the constant $b$ but the residue $A$ in (3) now becomes $2^{2 / 3} \pi^{2} \simeq 15.667$ instead of the previous $\pi^{2} / 2^{1 / 3} \simeq 7.8335$ associated with the original London equation, and fully agrees with the empirical residue of $15.7 \pm 0.6$, extracted by Cole [1] from high-pressure crystalline-branch data in ${ }^{3} \mathrm{He},{ }^{4} \mathrm{He}, \mathrm{H}_{2}$ and $\mathrm{D}_{2}$ systems. Moreover, this ML equation exhibits dramatically better agreement than the original London (L) equation with Green-function Monte Carlo (GFMC) [12 computer-simulation data points for both fluid and crystalline branches of the boson hard-sphere system.

A generalized London equation has also been proposed [13] for $N$-fermion hard-sphere systems with $\nu$ intrinsic degrees of freedom for each fermion. Here $\nu=2$ for, say, liquid ${ }^{3} \mathrm{He}$ or neutron matter, both constituent fermions of which have spin $1 / 2$, and $\nu=4$ for nuclear matter consisting of both neutrons and protons of spin $1 / 2$. As $\nu$ is essentially the maximum occupation in a given single-particle quantum state, it can be taken as infinite in the case of bosons. For fermions, two differences appear with respect to the boson London Formula: (a) unlike the boson case, the ground-state kinetic energy for fermions is nonzero and is added as a well-known [14 $\nu$-dependent leading term; and (b) the constant $b$ is allowed to be $\nu$-dependent, being replaced by

$$
b_{\nu}(\nu)=[(\nu-1) / \nu](b+1)-1
$$

which clearly approaches $b$ as $\nu \rightarrow \infty$. The latter form also ensures a $\nu$-independent energy at close-packing where, since the spheres can be labeled so that indistinguishability as well as particle statistics disappears, as expected in this classical limit. Substitution of $b_{\nu}$ for the constant $b$ in (1) gives a generalized form of the modified London equation $\left(\mathrm{ML}_{\nu}\right)$

$$
E / N=C_{\nu} \rho^{2 / 3}+\left(\frac{\nu-1}{\nu}\right) \frac{2 \pi \hbar^{2} c}{m} \frac{1}{\left(\rho^{-1 / 3}-\rho_{0}^{-1 / 3}\right)^{2}} \frac{1}{\left[\rho^{-1 / 3}+b_{\nu}(\nu) \rho_{0}^{-1 / 3}\right]}
$$

with

$$
C_{\nu} \equiv \frac{3 \hbar^{2}}{10 m}\left(\frac{6 \pi^{2}}{\nu}\right)^{2 / 3} \underset{\nu \rightarrow \infty}{\longrightarrow} 0 .
$$

For $\nu \rightarrow \infty, b(\nu) \rightarrow b$ according to (6), and (7) goes over into the boson case (11) because $C_{\nu}$ vanishes in this limit. The low-density limit of (7) is

$$
E / N \underset{\rho \rightarrow 0}{\longrightarrow} C_{\nu} \rho^{2 / 3}+\left(\frac{\nu-1}{\nu}\right) \frac{2 \pi \hbar^{2}}{m} \rho c
$$


where the second term on the rhs is the Lenz term for $\nu$-component fermions in 3D. On the other hand, for $\rho \rightarrow \rho_{0} \equiv \sqrt{2} / c^{3}$ one sees that (7) reduces to (3) as it should. In other words, hard-sphere fermions, bosons or "boltzons" must all closepack regularly at the same density. From this it follows that the residue for bosons or fermions is the same and equal to $2^{2 / 3} \pi^{2} \simeq 15.667$, in excellent agreement with the empirical Ref. [1] value of $15.7 \pm 0.6$.

For bosons, in addition to the Lenz term (2) for the low-density fluid branch, several higher-order corrections to the ground-state energy per particle have been derived using quantum field-theoretic many-boson perturbation theory [15] [16]. They give

$$
E / N=\frac{2 \pi \hbar^{2} \rho c}{m}\left\{1+C_{1}\left(\rho c^{3}\right)^{1 / 2}+C_{2} \rho c^{3} \ln \left(\rho c^{3}\right)+C_{3} \rho c^{3}+o\left(\rho c^{3}\right)\right\}
$$

for $\rho c^{3} \ll 1$, where $C_{1}=128 / 15 \sqrt{\pi}$ and $C_{2}=8(4 \pi / 3-\sqrt{3})$, but $C_{3}$ is an as yet unknown constant. Here, $c$ denotes the $\mathrm{S}$-wave scattering length for a general potential; for a hard-core potential it is just the hard-sphere diameter. The series is clearly not a pure power series expansion, and is at best an asymptotic series.

Similarly, for an $N$-fermion hard-sphere system the corresponding series is [17]

$$
\begin{aligned}
E / N & =\frac{3}{5} \frac{\hbar^{2} k_{F}^{2}}{2 m}\left\{1+C_{1}\left(k_{F} c\right)+C_{2}\left(k_{F} c\right)^{2}\right. \\
& +\left[C_{3} r_{0} / 2 c+C_{4} A_{1}(0) / c^{3}+C_{5}\right]\left(k_{F} c\right)^{3}+C_{6}\left(k_{F} c\right)^{4} \ln \left(k_{F} c\right) \\
& \left.+\left[C_{7} r_{0} / 2 c+C_{8} A_{0}^{\prime \prime}(0) / c^{3}+C_{9}\right]\left(k_{F} c\right)^{4}+o\left(k_{F} c\right)^{4}\right\}
\end{aligned}
$$

for $k_{F} c \ll 1$ and where the $C_{j}(j=1,2, \ldots, 9)$ are dimensionless coefficients depending on $\nu$; they are given in Ref. [18] for $\nu=2$ and $\nu=4$. The Fermi momentum $\hbar k_{F}$ is defined through the fermion-number density

$$
\rho \equiv N / \Omega=\nu k_{F}^{3} / 6 \pi^{2}
$$

with $\Omega$ the system volume, so that the Lenz term expressed in terms of $\rho$ is identical to the boson Lenz term apart from a factor of $(\nu-1) / \nu$ which is the average number of fermions the Pauli Principle allows a given fermion to interact with at the shortest possible range.

Unfortunately, both low-density expansions (10) and (11) lack accuracy at moderate to high densities, including the saturation (or equilibrium, zero-pressure) densities of liquid ${ }^{4} \mathrm{He}(\nu=\infty)$ [19] and liquid ${ }^{3} \mathrm{He}(\nu=2)$ or nuclear matter $(\nu=4)$. However, one can extrapolate the series for hard-sphere systems to physical and even to close-packing densities through the use of Padé [20] and/or a modest extension of these called the "tailing" 21] approximants. The so-called quantum thermodynamic (or van der Waals) perturbation theory (QTPT) [22, 23] has provided fairly accurate representations of the fluid branch of the equation of state of quantum hard-sphere systems [24], even beyond freezing (or, Kirkwood) phase transition densities, but without sufficient credibility as one approaches close packing. This is clear since one does not possess a single ground-state energy function with implicit information of both fluid and crystalline branches, with presumably different close-packing ultimate densities.

In Section 2 we discuss the double- (or second-order-) pole behavior for the equation-of-state fluid branch conceivably ending at random closest close-packing, instead of the regular close-packing at which the crystalline branch terminates; in Secs. 3 and 4 we construct analytical expressions for the fluid branches for hard-sphere bosons and fermions, respectively. Sec. 5 gives our conclusions.

\section{Double-pole conditions at close-packing}

We shall assume that the fluid branch of the hard-sphere equation of state will terminate not at the regular close-packing density $\rho_{0}$ but rather at the random closest close packing (rccp), sometimes called the Bernal, density $\rho_{\text {rccp }}$ (or "maximally random jammed" packing [25]). Its value was originally determined empirically [26] with actual ball-bearing packings. Near the density $\rho_{\text {rccp }}$ we expect, based on (3), that the energy for a hard sphere boson or fermion gas has the following behavior

$$
E / N \underset{\rho \rightarrow \rho_{r c c p}}{\longrightarrow} A\left(\hbar^{2} / 2 m\right)\left(\rho^{-1 / 3}-\rho_{r c c p}^{-1 / 3}\right)^{-2}
$$


with $A$ the residue which could be different for each system. Random close-packing densities range [27] from about $0.06 \rho_{0}$ to $0.86 \rho_{0} \equiv \rho_{\text {rccp }}$.

The derivative of (13) with respect to $\rho$ then tends asymptotically to

$$
\frac{d(E / N)}{d \rho} \underset{\rho \rightarrow \rho_{r c c p}}{\longrightarrow} \frac{A(2 / 3)\left(\hbar^{2} / 2 m\right)}{\left(\rho^{-1 / 3}-\rho_{r c c p}^{-1 / 3}\right)^{3} \rho^{4 / 3}}
$$

while

$$
\frac{d \ln (E / N)}{d \rho} \underset{\rho \rightarrow \rho_{\text {rccp }}}{\longrightarrow} \frac{2 / 3}{\left(\rho^{-1 / 3}-\rho_{r c c p}^{-1 / 3}\right) \rho^{4 / 3}}
$$

is residue independent. We shall assume that $A$ is the same for boson as for fermion hard spheres and that their rccp density is likewise identical since at closest close-packing the particles become localized by definition, enabling one to formally label each particle; this makes them distinguishable thus rendering (quantum) statistics irrelevant. Note that the pressure $P=\rho^{2}[d(E / N) / d \rho]$ from (14) also diverges as $\rho \longrightarrow \rho_{\text {rccp }}$, as expected.

\section{Boson hard-sphere fluid}

In order to extrapolate the low-density series (10) to higher densities we start by writing it as

$$
E / N=\frac{2 \pi \hbar^{2}}{m} \rho c e_{0}(x)
$$

where $x \equiv\left(\rho c^{3}\right)^{1 / 2}$ and

$$
e_{0}(x) \equiv 1+C_{1} x+C_{2} x^{2} \ln x^{2}+C_{3} x^{2}+O\left(x^{3} \ln x^{2}\right)
$$

for $x \ll 1$. Alternatively, one can rewrite this series as

$$
e_{0}^{-1 / 2}(x)=1+K_{1} x+K_{2} x^{2} \ln x^{2}+K_{3} x^{2}+O\left(x^{3} \ln x^{2}\right)
$$

where the $K_{i}$ 's are expressible in terms of the $C_{i}$ 's. As $C_{3}$ is to date unknown, consequently $K_{3}$ is also unknown. Values of the $C_{i}$ 's and $K_{i}$ 's are given in Table1. We analyze the series $e_{0}^{-1 / 2}(x)$ instead of the series $e_{0}(x)$ to ensure that any zeros in its extrapolants, say $\epsilon_{0}^{-1 / 2}(x)$, are double (or second-order) poles in the energy as one expects at any kind of close packing. The extrapolants are generated as a quotient of two polynomials such that on expansion one recovers the first terms of the original series. Series (18) with three terms beyond unity has twelve extrapolants correctly generated in Ref. 28] but fitted there to erroneous (i.e., to one-half the correct values) GFMC data points [12]. Adjusting various extrapolants 24] to best-fit the four known GFMC data points ensures a good value for the unknown coefficient $K_{3}$ in (18). The extrapolant labeled "XI (bosons)" in Fig. 2 of Ref. 24] had the least mean-square deviation with respect to the four GFMC fluid-branch data points. Therefore, we adopt it as our best initial extrapolant. The ground-state energy per particle for boson hard spheres was thus represented (symbol $\doteq$ ) by

$$
E / N \doteq \frac{2 \pi \hbar^{2}}{m} \rho c \epsilon_{0}(\rho)
$$

with $K_{3} \simeq-27.956$. However, as diffusion Monte Carlo (DMC) calculations became available [29] spanning a wider range of densities in the fluid region than GFMC data, we realized that although our expression XI $(x)$ in Eq. (17) of Ref. 24] agrees

\begin{tabular}{||c|c|c|c||}
\hline \hline Bosons $(\nu=\infty)$ & $i=1$ & 2 & 3 \\
\hline$C_{i}$ & 4.81441778 & 19.65391518 & "73.296" \\
\hline$K_{i}$ & -2.40720889 & -9.826957589 & "-27.956" \\
\hline \hline
\end{tabular}

Table 1: Coefficients $C_{i}$ and $K_{i}$ for bosons appearing in (17) and (18), respectively. Numbers in quotation marks are determined as indicated in text. 
well with DMC and GFMC data around the freezing transition, its disagreement with the DMC data at low to intermediate densities suggested the possibility of improving the extrapolant. As will be seen, the new extrapolant $\epsilon_{0}^{-1 / 2}$ predicts a random closet close-packing (rccp) density $\rho_{\text {rccp }} / \rho_{0} \simeq 0.776$ which is only about $10 \%$ below the classical hard-spheres empirical [26] rccp value $\simeq 0.86$ mentioned before and also assumed to be the ultimate rccp density for quantum hard-sphere fluids.

In order to improve the fluid-branch expression of Ref. 24] for low to intermediate densities we use the two double-pole conditions (13) and (14) which lead to the following conditions on the extrapolant $\epsilon_{0}(x)$ to be used in (19), namely

$$
\epsilon_{0}=\frac{m E}{N 2 \pi \hbar^{2} \rho c} \quad \underset{\rho \rightarrow \rho_{r c c p}}{\longrightarrow} \quad \frac{A}{4 \pi \rho c}\left(\rho^{-1 / 3}-\rho_{r c c p}^{-1 / 3}\right)^{-2}
$$

This is equivalent to

The condition (14) gives

$$
\epsilon_{0}^{-1 / 2}(\rho) \underset{\rho \rightarrow \rho_{\text {rccp }}}{\longrightarrow}[A / 4 \pi \rho c]^{-1 / 2}\left(\rho^{-1 / 3}-\rho_{r c c p}^{-1 / 3}\right) \underset{\rho \rightarrow \rho_{\text {rccp }}}{\longrightarrow} \quad 0 .
$$

$$
\frac{d\left(\epsilon_{0}^{-1 / 2}\right)}{d \rho} \underset{\rho \rightarrow \rho_{\text {rccp }}}{\longrightarrow}-(1 / 3)[A / 4 \pi c]^{-1 / 2} \rho_{r c c p}^{-5 / 6}
$$

Strictly, any log term should be accompanied by a constant, if known, because the scaling of $\rho$ by $c^{3}$ is arbitrary. We thus propose the representation of $e_{0}(x)$ in (17) as given by

$$
e_{0}^{-1 / 2}(x) \doteq \frac{1+K_{1} x+\beta x^{2}+\gamma x^{3}}{1-K_{2} x^{2} \ln x^{2}+\alpha x^{2}} \equiv \epsilon_{0 B}^{-1 / 2}(x)
$$

where $\alpha, \beta$ and $\gamma$ are to be determined from (20) and (21) and by fitting both DMC 29] and GFMC ([12], Table I) data. In this approximant the terms in $x^{2} \ln x$ and $x^{2}$ are kept together. Condition (20) applied to (22) gives

$$
1+K_{1} x_{r c c p}+\beta x_{r c c p}^{2}+\gamma x_{r c c p}^{3}=0 .
$$

The second condition (21) can be rewritten as

$$
\left.\frac{d\left(\epsilon_{0 B}^{-1 / 2}\right)}{d x} \frac{d x}{d \rho}\right|_{x=x_{r c c p}}=\frac{K_{1}+2 \beta x_{r c c p}+3 \gamma x_{r c c p}^{2}}{1-K_{2} x_{r c c p}^{2} \ln x_{r c c p}^{2}+\alpha x_{r c c p}^{2}} \frac{c^{3}}{2 x_{r c c p}}=-(1 / 3)[A / 4 \pi c]^{-1 / 2} \rho_{r c c p}^{-5 / 6} .
$$

Substituting (23) in the last equation we obtain $\beta$ in terms of $\alpha$, namely

$$
-\beta x_{r c c p}^{2}=3+2 K_{1} x_{r c c p}-[A / 4 \pi]^{-1 / 2} \frac{2}{3} x_{r c c p}^{1 / 3}\left(1-K_{2} x_{r c c p}^{2} \ln x_{r c c p}^{2}+\alpha x_{r c c p}^{2}\right) .
$$

Now substituting (25) in (23) we arrive at

$$
\gamma x_{r c c p}^{3}=2+K_{1} x_{r c c p}-[A / 4 \pi]^{-1 / 2} \frac{2}{3} x_{r c c p}^{1 / 3}\left(1-K_{2} x_{r c c p}^{2} \ln x_{r c c p}^{2}+\alpha x_{r c c p}^{2}\right) .
$$

Introducing (25) and (26) in (22), we get

$$
\begin{aligned}
\epsilon_{0 B}^{-1 / 2}(x)=[1 & +K_{1} x+\left(x / x_{r c c p}\right)^{2}\left\{-3-2 K_{1} x_{r c c p}\right\}+\left(x / x_{r c c p}\right)^{3}\left\{2+K_{1} x_{r c c p}\right\}+[A / 4 \pi]^{-1 / 2} \frac{2}{3} x_{r c c p}^{1 / 3} \\
& \left.\times\left(1-K_{2} x_{r c c p}^{2} \ln x_{r c c p}^{2}+\alpha x_{r c c p}^{2}\right)\left\{\left(x / x_{r c c p}\right)^{2}-\left(x / x_{r c c p}\right)^{3}\right\}\right]\left[1-K_{2} x^{2} \ln x^{2}+\alpha x^{2}\right]^{-1}
\end{aligned}
$$

from which after some algebra one obtains a single equation for $\alpha$, namely

$$
\begin{aligned}
& {\left[\epsilon_{0 B}^{-1 / 2}(\alpha, A, x)\right]\left(1-K_{2} x^{2} \ln x^{2}\right)-1-K_{1} x+\left(x / x_{r c c p}\right)^{2}\left\{3+2 K_{1} x_{r c c p}\right\}} \\
& -[A / 4 \pi]^{-1 / 2} \frac{2}{3} x_{r c c p}^{1 / 3}\left(1-K_{2} x_{r c c p}^{2} \ln x_{r c c p}^{2}\right)\left\{\left(x / x_{r c c p}\right)^{2}-\left(x / x_{r c c p}\right)^{3}\right\}-\left(x / x_{r c c p}\right)^{3}\left\{2+K_{1} x_{r c c p}\right\} \\
& =\alpha\left\{x_{r c c p}^{2}[A / 4 \pi]^{-1 / 2} \frac{2}{3} x_{r c c p}^{1 / 3}\left[\left(x / x_{r c c p}\right)^{2}-\left(x / x_{r c c p}\right)^{3}\right]-x^{2}\left[\epsilon_{0 B}^{-1 / 2}(\alpha, A, x)\right]\right\} .
\end{aligned}
$$

where we have explicitly written the dependence of $\epsilon_{0 B}^{-1 / 2}(x)$ on $\alpha$ and $A$. To determine $\alpha$ from the DMC [29] and/or GFMC data we must calculate the values $\alpha_{i}^{D M C}$ [from (28) after replacing $\epsilon_{0 B}^{-1 / 2}(\alpha, A, x)$ by the $\epsilon_{0-D M C}^{-1 / 2}\left(x_{i}^{D M C}\right)$ obtained from 
(19) as $\left(2 \pi \hbar^{2} \rho c N / E m\right)^{1 / 2}$ with $E / N$ the energy from DMC calculations] for each $x_{i}^{D M C}$ for $i=1,2, \cdots N$ values, and then minimizes $\sum_{i=1}^{N}\left(\alpha_{i}^{D M C}-\alpha\right)^{2}$ by imposing

$$
\frac{d}{d \alpha} \sum_{i=1}^{N}\left(\alpha_{i}^{D M C}-\alpha\right)^{2}=0
$$

which gives

$$
\alpha=\sum_{i=1}^{N} \alpha_{i}^{D M C} / N
$$

Since the fluid branch GFMC data are a subset of DMC data, we have used these to calculate $\alpha$ here, determining $A$ in the next step. For residue $A$ fixed at $2^{2 / 3} \pi^{2} \simeq 15.667$ as described below (5), we obtain an optimal $\alpha \simeq 114.282$ which from (25) and (26) leads to $\beta \simeq 74.0891$ and $\gamma \simeq-65.9475$. The curve then corresponding to (22) is labeled B1 in Fig. 1 .

Alternatively, if we allow the residue $A$ to be free one may ask for a solution minimizing $\sum_{i=1}^{N}\left[\epsilon_{0-D M C}^{-1 / 2}\left(x_{i}^{D M C}\right)-\right.$ $\left.\epsilon_{0 B}^{-1 / 2}\left(\alpha, A, x_{i}^{D M C}\right)\right]^{2}$ with respect $\alpha$ and $A$, i.e.,

$$
\frac{d}{d \alpha} \sum_{i=1}^{N}\left[\epsilon_{0-D M C}^{-1 / 2}\left(x_{i}^{D M C}\right)-\epsilon_{0 B}^{-1 / 2}\left(\alpha, A, x_{i}^{D M C}\right)\right]^{2}=-\sum_{i=1}^{N} 2\left[\epsilon_{0-D M C}^{-1 / 2}\left(x_{i}^{D M C}\right)-\epsilon_{0 B}^{-1 / 2}\left(\alpha, A, x_{i}^{D M C}\right)\right] \frac{d}{d \alpha} \epsilon_{0 B}^{-1 / 2}\left(\alpha, A, x_{i}^{D M C}\right)=0
$$

or

$$
\sum_{i=1}^{N} 2\left[\epsilon_{0-D M C}^{-1 / 2}\left(x_{i}^{D M C}\right)-\epsilon_{0 B}^{-1 / 2}\left(\alpha, A, x_{i}^{D M C}\right)\right] \frac{-Y\left(\alpha, A, x_{i}\right) x^{2}+(A / 4 \pi)^{-1 / 2} \frac{2}{3} x_{r c c p}^{1 / 3} x_{r c c p}^{2}\left[\left(x / x_{r c c p}\right)^{2}-\left(x / x_{r c c p}\right)^{3}\right]}{1-K_{2} x^{2} \ln x^{2}+\alpha x^{2}}=0
$$

as well as of

$$
\frac{d}{d A} \sum_{i=1}^{N}\left[\epsilon_{0-D M C}^{-1 / 2}\left(x_{i}^{D M C}\right)-\epsilon_{0 B}^{-1 / 2}\left(\alpha, A, x_{i}^{D M C}\right)\right]^{2}=-\sum_{i=1}^{N} 2\left[\epsilon_{0-D M C}^{-1 / 2}\left(x_{i}^{D M C}\right)-\epsilon_{0 B}^{-1 / 2}\left(\alpha, A, x_{i}^{D M C}\right)\right] \frac{d}{d A} \epsilon_{0 B}^{-1 / 2}\left(\alpha, A, x_{i}^{D M C}\right)=0
$$

or

$$
\sum_{i=1}^{N} 2\left[\epsilon_{0-D M C}^{-1 / 2}\left(x_{i}^{D M C}\right)-\epsilon_{0 B}^{-1 / 2}\left(\alpha, A, x_{i}^{D M C}\right)\right] \frac{(A / 4 \pi)^{-3 / 2} \frac{1}{12 \pi} x_{r c c p}^{1 / 3}\left(1-K_{2} x_{r c c p}^{2} \ln x_{r c c p}^{2}+\alpha x_{r c c p}^{2}\right)\left[\left(x / x_{r c c p}\right)^{2}-\left(x / x_{r c c p}\right)^{3}\right]}{1-K_{2} x^{2} \ln x^{2}+\alpha x^{2}}=0 .
$$

Under the two conditions (29) and (30) we find an optimal $A \simeq 11.8715$ and an optimal $\alpha \simeq 169.516$, leading to $\beta \simeq 124.1$ and $\gamma \simeq-111.296$. This procedure gives the curve labeled B2 in Fig. 1. Note that the residue 11.8715 is now being associated with the random closest close-packing (rccp) density $0.86 \rho_{0}$ of hard spheres. This value of $A$ is somewhat smaller than the residue 15.667 at regular close-packing density $\rho_{0}$, though still within the rigorous range stated in (44). Figure 2 compares the previous fluid branch expression XI $(x)$, Eq. (17) of Ref. 24], with the present extrapolant (22) labeled B2, both as full curves. The dashed curve is the modified London (ML) formula (11) that connects smoothly with the crystalline branch. Open circles and squares are GFMC data for fluid and crystalline branches, respectively. Dots represent DMC [29] data spanning a wider range of densities in the fluid region than the GFMC data. The new expression B2 shows dramatically better agreement with DMC data for intermediate densities, as well as agreeing well with both DMC and GFMC data around the freezing transition mentioned in Table I of Ref. [12. Fig. 3 is an enlargement of Fig. 2 at low densities to show the remarkable agreement of $\mathrm{B} 2$ with the DMC data.

\section{Fermion hard-sphere fluid branch}

The ground-state energy per particle for fermion hard-sphere fluids (11) can be written as

$$
E / N=\frac{3}{5} \frac{\hbar^{2} k_{F}^{2}}{2 m} e_{0}(x), \quad x \equiv k_{F} c
$$




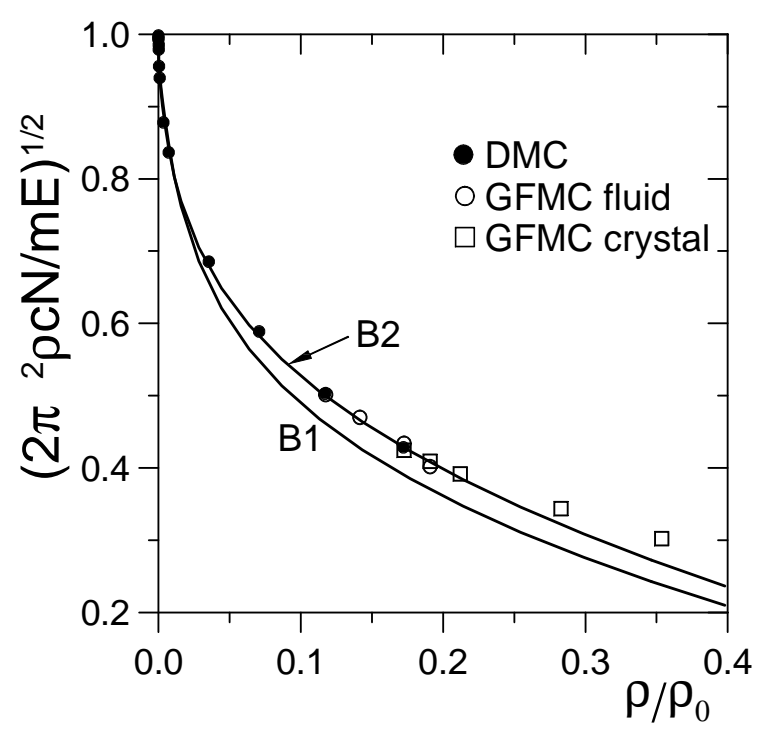

Figure 1: The quantity $\epsilon_{0}^{-1 / 2}=\sqrt{2 \pi \hbar^{2} \rho c N / m E}=\left[1-\left(\rho / \rho_{0}\right)^{1 / 3}\right] \sqrt{1+b\left(\rho / \rho_{0}\right)^{1 / 3}}$ as a function of $x / x_{0}$ for boson hard sphere systems: B1 and B2 refer to (22) and (24) with $A \simeq 15.7$ and $A \simeq 11.9$, respectively. Larger dots are GFMC fluid data and smaller dots refer to DMC (fluid) calculations.

with

$$
\begin{aligned}
e_{0}(x) \equiv 1 & +C_{1} x+C_{2} x^{2}+\left(C_{3} / 3+C_{4} / 3+C_{5}\right) x^{3} \\
& +C_{6} x^{4} \ln x+\left(C_{7} / 3-C_{8} / 3+C_{9}\right) x^{4}+o\left(x^{4}\right)
\end{aligned}
$$

for $x \equiv k_{F} c \ll 1, \rho \equiv N / \Omega=\nu k_{F}^{3} / 6 \pi^{2}$ being the number of fermions $N$ in the enclosed volume $\Omega$. We shall examine both $\nu=2$ (corresponding to liquid ${ }^{3} \mathrm{He}$ and neutron matter) and $\nu=4$ (corresponding to nuclear matter).

\subsection{Fermions with $\nu=2$}

For $\nu=2, C_{6}=0$ [17] so that (32) simplifies to the pure power series

$$
e_{0}(x)=1+D_{1} x+D_{2} x^{2}+D_{3} x^{3}+D_{4} x^{4}+o\left(x^{4}\right)
$$

where the $C_{i}$ 's have been determined in terms of the $D_{i}$ 's. As in the boson case, instead of $e_{0}(x)$ we consider the series

$$
e_{0}^{-1 / 2}(x)=1+F_{1} x+F_{2} x^{2}+F_{3} x^{3}+F_{4} x^{4}+F_{5} x^{5}+o\left(x^{5}\right)
$$

where the $F_{i}$ 's depend algebraically on the $D_{i}$ 's in a simple manner, $F_{5}$ being unknown. Values of $D_{i}$ and $F_{i}$ are given in Table 2 We use this simple power series to construct the usual Padé extrapolants. The approximants to (34) with four terms beyond the trivial unity were analyzed in Ref. [30] where it was concluded that the best approximant was the Padé $[0 / 4](x)$. However, this function does not have a zero in the region of physical interest, i.e., $0 \leq \rho / \rho_{0} \leq 1$, which implies $0 \leq x \equiv k_{F} c \leq 3.47$ since $\rho=k_{F}^{3} / 3 \pi^{2}$. Accordingly, the energy does not manifest a close-packing density as it should. This deficiency made it advisable to introduce the fifth term $F_{5} x^{5}$ in (34). Although in Fig. 1 of Ref. 31] only five of the six two-point Padé approximants $[L / / M](x)$ with $L+M=5$, are shown, all six approximants were analyzed here to adjust $F_{5}$ so as to ensure a zero associated with a random close-packing in the physical region. The approximant $\epsilon_{0}(x)$ and the position of its zero were chosen in such way that the QTPT applied in Ref. [30] to calculate the ground-state energy of ${ }^{3} \mathrm{He}$ with

the Aziz interatomic potential 32] reproduces the corresponding GFMC 34 data. (In this treatment, the Aziz potential 


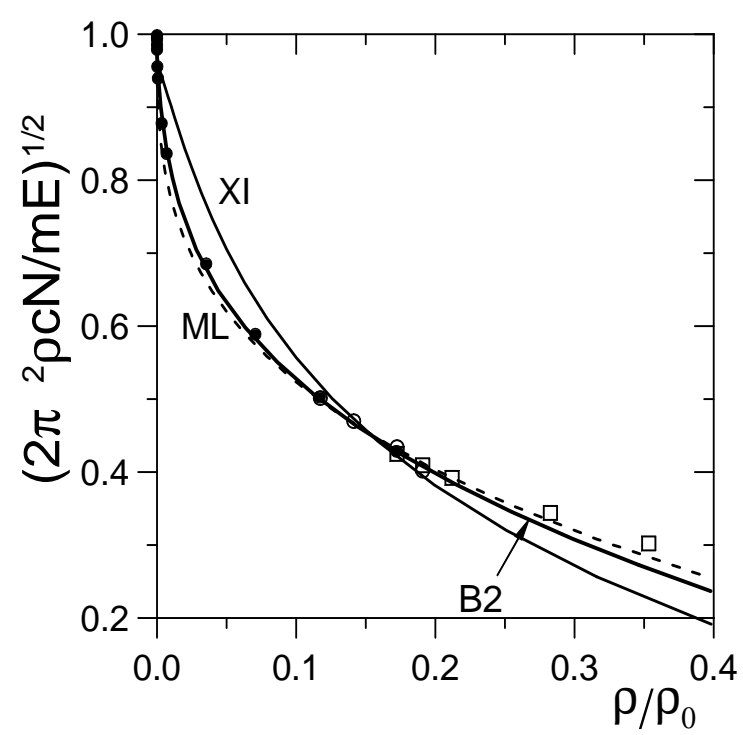

Figure 2: The quantity $\epsilon_{0}^{-1 / 2}=\sqrt{2 \pi \hbar^{2} \rho c N / m E}=\left[1-\left(\rho / \rho_{0}\right)^{1 / 3}\right] \sqrt{1+b\left(\rho / \rho_{0}\right)^{1 / 3}}$ as a function of $\rho / \rho_{0}$ for the boson hard sphere system: XI is the fluid branch approximant of Ref. [24], Fig. 2; B2 refers to (22) and (24) with $A \simeq 11.9$; ML is the modified London formula (11). Open circles and squares are GFMC data for the fluid and crystalline branches, respectively, and dots are DMC data points.

was decomposed via the well-known Barker-Henderson (BH) [33] scheme as described in Ref. [30.) Eventually, the best extrapolant was found to be the two-point Padé approximant

$$
e_{0}^{-1 / 2}(x) \doteq[3 / / 2](x) \equiv \frac{N_{0}+N_{1} x+N_{2} x^{2}+N_{3} x^{3}}{M_{0}+M_{1} x+M_{2} x^{2}} \equiv \epsilon_{0}^{-1 / 2}(x)
$$

where

$$
\begin{aligned}
& N_{0}=F_{2} F_{4}-F_{3}^{2} \\
& N_{1}=F_{4}\left(F_{3}+F_{1} F_{2}\right)-F_{2} F_{5}-F_{1} F_{3}^{2} \\
& N_{2}=\left(F_{3}-F_{1} F_{2}\right) F_{5}-F_{4}^{2}+\left(F_{1} F_{3}+F_{2}^{2}\right) F_{4}-F_{2} F_{3}^{2} \\
& N_{3}=\left(F_{1} F_{3}-F_{2}^{2}\right) F_{5}-F_{1} F_{4}^{2}+2 F_{2} F_{3} F_{4}-F_{3}^{3} \\
& M_{0}=F_{2} F_{4}-F_{3}^{2} \\
& M_{1}=F_{3} F_{4}-F_{2} F_{5} \\
& M_{2}=F_{3} F_{5}-F_{4}^{2} .
\end{aligned}
$$

The extrapolant (35) satisfies $[3 / / 2](x=3.13)=0$. Hence the ground-state energy per fermion for $\nu=2$ becomes

$$
E / N \doteq \frac{3}{5} \frac{\hbar^{2} k_{F}^{2}}{2 m}\{[3 / / 2](x)\}^{-2}
$$

\begin{tabular}{||c|c|c|c|c|c||}
\hline \hline$\nu=2$ & $i=1$ & 2 & 3 & 4 & 5 \\
\hline$D_{i}$ & 0.353678 & 0.185537 & 0.384145 & -0.024700 & “- $0.265544 "$ \\
\hline$F_{i}$ & -0.176833 & -0.045863 & -0.156677 & 0.109672 & "0.130830" \\
\hline \hline
\end{tabular}

Table 2: Coefficients $D_{i}$ and $F_{i}$ for $\nu=2$ appearing in (33) and (34), respectively. Numbers in quotation marks were determined as indicated in text. 


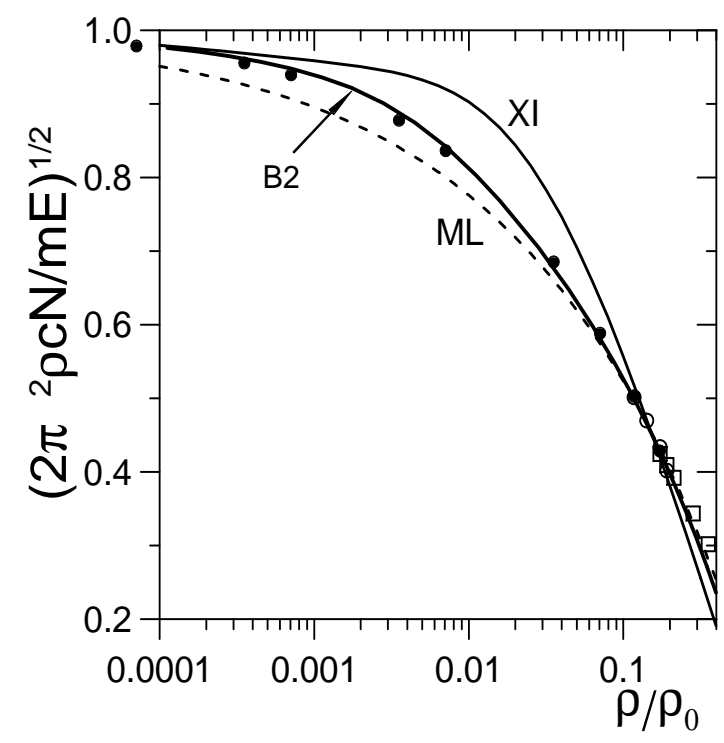

Figure 3: Enlargement of Fig. [2] at low densities.

with a random closest close-packing density $\rho_{\text {rccp }} / \rho_{0}=0.732$ only $15 \%$ smaller than the empirical [26] value $\rho_{\text {rccp }} / \rho_{0} \simeq 0.86$. The coefficient $F_{5}$ is listed in Table 2 in quotation marks. In Fig. 4 we show the expression

$$
\begin{aligned}
& \epsilon_{0}^{-1 / 2}=\left[3 \hbar^{2}\left(6 \pi^{2} \rho / \nu\right)^{2 / 3} N / 10 m E\right]^{1 / 2}=1+ \\
& {[20 \pi(\nu-1) / 3 \nu]\left(2^{1 / 4} \nu / 6 \pi^{2}\right)^{2 / 3}\left\{\left[\left(\rho / \rho_{0}\right)^{-1 / 3}-1\right]^{2}\left[\left(\rho / \rho_{0}\right)^{-1 / 3}-b(\nu)\right]\left(\rho / \rho_{0}\right)^{2 / 3}\right\}^{-1}}
\end{aligned}
$$

as a function of $\rho / \rho_{0}$ for fermion hard spheres. Here $b(\nu)$ is as defined in (66). For $\nu=2$ the fluid branch [3//2] (full curve) given by (35) is close to the Ladder [35] (open squares), the variational Fermi hypernetted chain (VFHNC) [36] (plus-sign marks), and the so-called L-expansion data [37, 38, (open triangles). Fig. 4 shows good agreement over the entire range of available data.

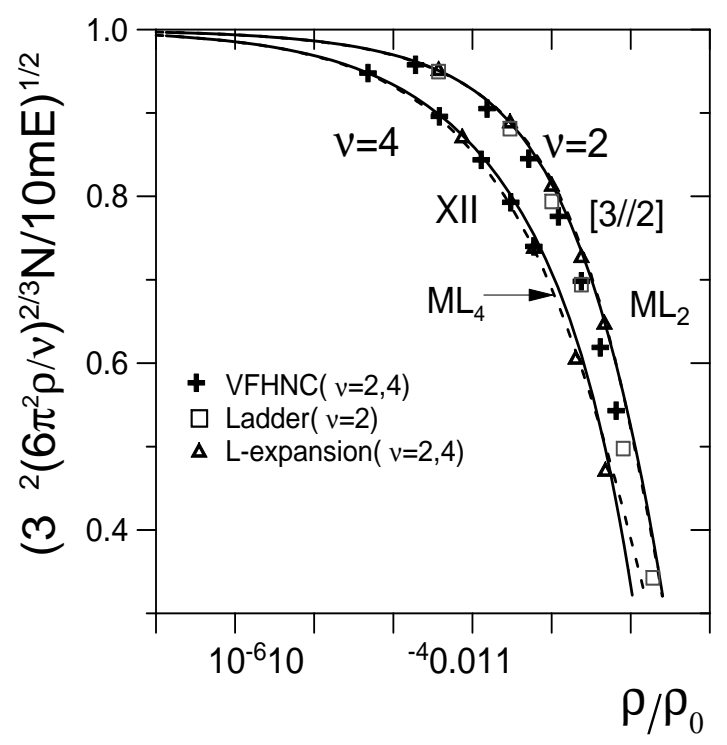

Figure 4: The expression (37) as function of $\rho / \rho_{0}$ for fermion hard spheres with $\nu=2$ labeled $[3 / / 2]$ and with $\nu=4$ labeled XII (full curves). Dashed curves are the corresponding modified London $\mathrm{ML}_{\nu}$ formulae, but note that the $\mathrm{ML}_{2}$ dashed curve almost coincides with the full curve $[3 / / 2]$. 
In order to improve the many-fermion ground-state energy equation of state we include the next term in (34), i.e., $F_{6} x^{6}$, which is then used to generate all Padé approximants of order six to the series $\epsilon_{0}^{-1 / 2}(x)$. The lack of a logarithmic term $x^{4} \ln x$ is due to the Pauli principle [17. Such a term arises when there are three independent hole lines. But for $\nu=2$ there can be at most two lines of the same spin. Thus the Pauli principle reduces the size of the term by a factor of the density. We thus expect the first such term for $\nu=2$ to be $O\left(x^{7} \ln x\right)$. The unknown coefficients $F_{6}$ and $F_{5}$ are determined from the two double-pole conditions (13) and (14), which become

$$
\epsilon_{0}^{-1 / 2}(x) \quad \underset{\rho \rightarrow \rho_{r c c p}}{\longrightarrow} \quad\left(1-x / x_{r c c p}\right)\left[5 A / 3\left(3 \pi^{2}\right)^{2 / 3}\right]^{-1 / 2}
$$

and

$$
\epsilon_{0}(x)+\frac{x}{2} \frac{d \epsilon_{0}(x)}{d x} \quad \underset{\rho \rightarrow \rho_{r c c p}}{\longrightarrow} \quad \frac{5 A / 3\left(3 \pi^{2}\right)^{2 / 3}}{\left(1-x / x_{r c c p}\right)^{3}}
$$

with $A \simeq 15.667$.

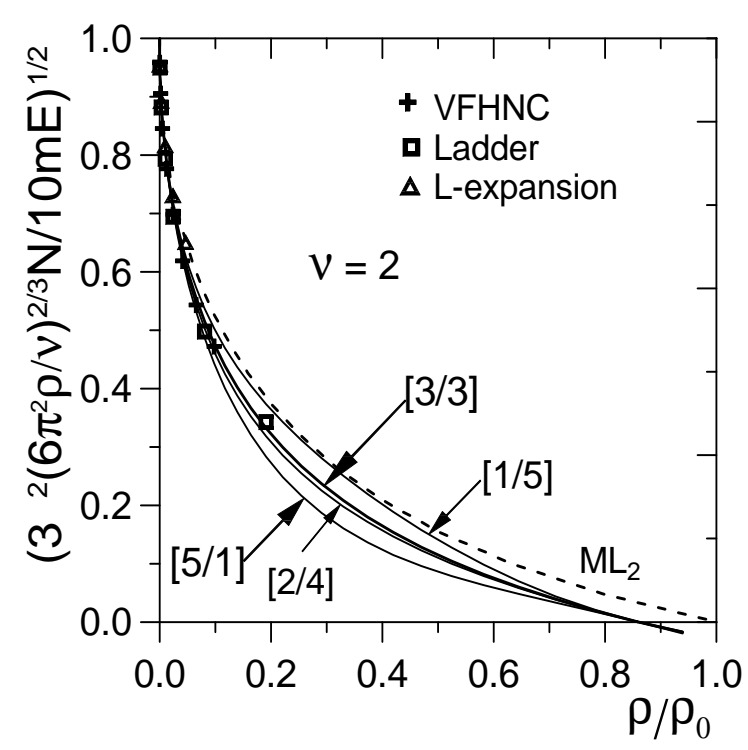

Figure 5: Improved extrapolants for the many-fermion hard-sphere gas with $\nu=2$.

For each Padé approximant of order six we determined $F_{6}$ and $F_{5}$ as shown in Table 3 Approximants [4/2] and [0/6] did not exhibit the double-pole conditions. The other four approximants are plotted in Fig. 5 together with the Ladder [35] (open squares), the variational Fermi hypernetted chain (VFHNC) 36] (plus-sign marks), and the L-expansion [37] (open triangles) data for $\nu=2$, from which we conclude that the approximant $[3 / 3](x)$ is the best. Fig. 6 is a semi-log enlargement of Fig. 5. In Fig. 7 we compare both the new improved expression [3/3] $(x)$ and the previous best energy expression, i.e, the two-point Padé approximant [3//2](x) reported in Ref. [24] and supported by Ladder, VFHNC and L-expansion data. 


\begin{tabular}{||c|c|c||}
\hline \hline Padé & F5 & F6 \\
\hline$[5 / 1]$ & -0.0272548 & 0.0038205 \\
\hline$[4 / 2]$ & -.20 & no solution \\
\hline$[3 / 3]$ & -0.0130625 & 0.0039120 \\
\hline$[2 / 4]$ & -0.0395076 & 0.0415222 \\
\hline$[1 / 5]$ & -0.0115902 & 0.01887153 \\
\hline$[0 / 6]$ & -0.1276 & no solution \\
\hline \hline
\end{tabular}

Table 3: The $F_{5}$ and $F_{6}$ coefficients for $\nu=2$ that follow from conditions (38) and (39) for all sixth-order Padé approximants with residue $A \simeq 15.667$ and random closest close-packing density $\rho_{r c c p} \equiv 0.86 \rho_{0}$.

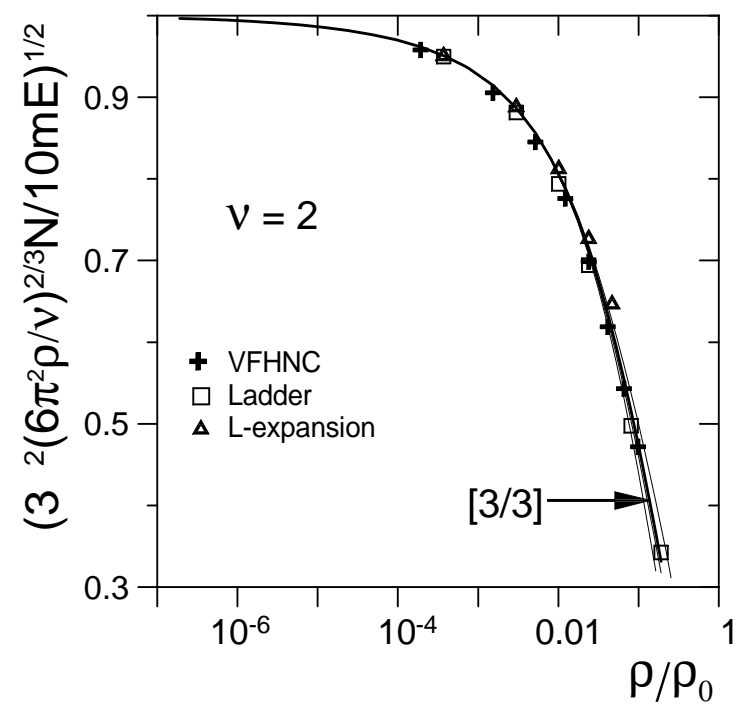

Figure 6: Enlargement of Fig. [5 at low densities.

\subsection{Fermions with $\nu=4$}

For fermions with $\nu=4(32)$ becomes

$$
e_{0}(x)=1+D_{1} x+D_{2} x^{2}+D_{3} x^{3}+D_{4} x^{4} \ln x+D_{5} x^{4}+o\left(x^{4}\right)
$$

for $x \equiv k_{F} c \ll 1$ and we recall that $\rho=\nu k_{F}^{3} / 6 \pi^{2}$. As for bosons or for fermions with $\nu=2$, we analyze

$$
e_{0}^{-1 / 2}(x)=1+F_{1} x+F_{2} x^{2}+F_{3} x^{3}+F_{4} x^{4} \ln x+F_{5} x^{4}+o\left(x^{4}\right)
$$

with all $F_{i}(i=1,2,3,4)$ known. Values of $D_{i}$ and $F_{i}$ are given in Table 4. Unlike the $\nu=2$ case, this series is not a pure power series as it contains logarithmic terms. Its so-called "tailing" 21] approximants are given in Table III of Ref. 28. Of all the possible approximants using only the known coefficients, only the forms II and XII are free from flaws and have residues within the bounds (4). Of these two forms, II has a residue less than that predicted in Ref. 77. Hence we chose form XII, which is plotted in Fig. 团as the full curve labeled XII.

In this case $E / N$ can be written as

$$
E / N=\frac{3 \hbar^{2} k_{F}^{2}}{10 m} \epsilon_{0}(x)
$$

where the series (41) is represented as

$$
e_{0}^{-1 / 2}(x) \doteq \operatorname{XII}(x) \equiv \frac{1+\left(F_{1}-F_{3} / F_{2}\right) x+\left(F_{2}-F_{1} F_{3} / F_{2}\right) x^{2}}{1-\left(F_{3} / F_{2}\right) x-F_{4} x^{4} \ln x} \equiv \epsilon_{0}^{-1 / 2}(x) .
$$




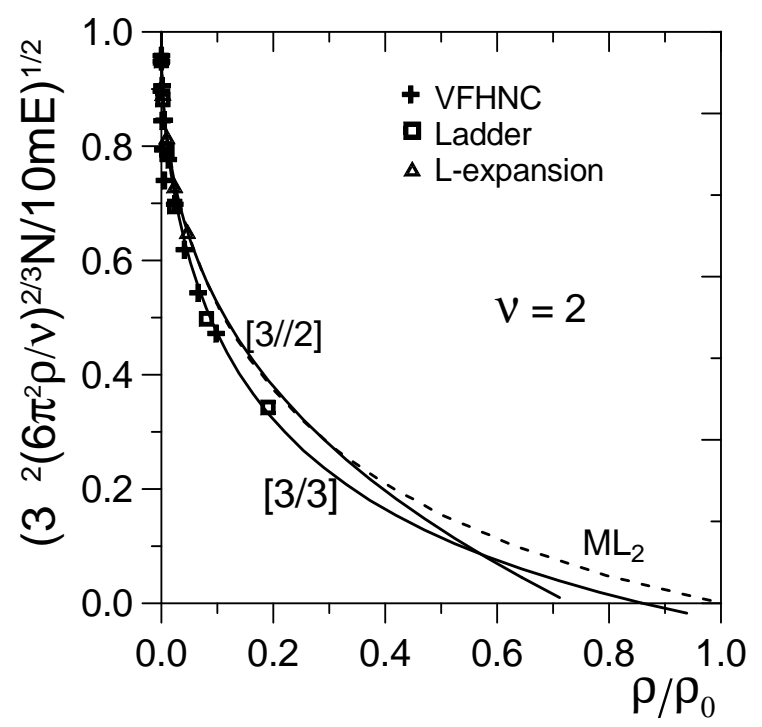

Figure 7: Comparison of quantity (37) as function of $\rho / \rho_{0}$ for many-fermion hard spheres with $\nu=2$, for the the previously best approximant [3//2](x) [24] and the new improved one [3/3](x), full curves. Dashed curve is modified London formula.

\begin{tabular}{||c|c|c|c|c||}
\hline \hline$\nu=4$ & $i=1$ & 2 & 3 & 4 \\
\hline$D_{i}$ & 1.061033 & 0.556610 & 1.300620 & -1.408598 \\
\hline$F_{i}$ & -0.530517 & 0.143867 & -0.5806558 & -0.704299 \\
\hline \hline
\end{tabular}

Table 4: Coefficients $D_{i}$ and $F_{i}$ for $\nu=4$ appearing in (40) and (41), respectively.

We also plot the corresponding VFHNC data (plus-sign marks) and L-expansion data (open triangles). In terms of energy, our results are slightly below the VFHNC points, with agreement improving at lower densities. On the other hand, the XII approximant lies just above the L-expansion data over the range of densities where data are available.

In order to improve the $\nu=4$ many-fermion hard-sphere ground-state energy equation of state, the energy series (11) was written as

$$
\begin{aligned}
\frac{E}{N}-\frac{3}{5} \frac{\hbar^{2} k_{F}^{2}}{2 m}=\frac{3}{5} \frac{\hbar^{2} k_{F}^{3} c}{2 m} e_{0}(x)=\quad & \frac{3}{5} \frac{\hbar^{2} k_{F}^{3} c}{2 m}\left[D_{1}+D_{2} x+D_{3} x^{2}+D_{4} x^{3} \ln x+D_{5} x^{3}\right. \\
& \left.+D_{6} x^{4} \ln x+D_{7} x^{4}+\cdots\right]
\end{aligned}
$$

where $x=k_{F} c$ and $\rho=\nu k_{F}^{3} / 6 \pi^{2}$. The suggested representation for $e_{0}(x)$ here is

$$
e_{0}(x)=D_{1}+D_{2} x+D_{3} x^{2}+D_{4} x^{3} \ln x+D_{5} x^{3}+D_{6} x^{4} \ln x+D_{7} x^{4}+\cdots
$$

which leads to

$$
e_{0}(x)^{-1 / 2}=F_{1}+F_{2} x+F_{3} x^{2}+F_{4} x^{3} \ln x+F_{5} x^{3}+F_{6} x^{4} \ln x+F_{7} x^{4}+\cdots
$$

with $D_{1}$ to $D_{4}$ known and equal to the values given in the Table 4 . The coefficients $F_{1}$ to $F_{4}$ are different from those in Table 4 , but they are derived simply from the $D_{i}$ 's and so are also known. They are

$$
\begin{gathered}
F_{1}=1 / \sqrt{D_{1}} ; \quad F_{2}=-D_{2} / 2 D_{1}^{3 / 2} ; \quad F_{3}=\left(3 D_{2}^{2}-4 D_{1} D_{3}\right) / 8 D_{1}^{5 / 2} ; \quad F_{4}=-8 D_{1}^{2} D_{4} / 16 D_{1}^{7 / 2} ; \\
F_{5}=\left(-5 D_{2}^{3}+12 D_{1} D_{2} D_{3}-8 D_{1}^{2} D_{5}\right) / 16 D_{1}^{7 / 2} ; \quad F_{6}=32 D_{1}^{2}\left(3 D_{2} D_{4}-2 D_{1} D_{6}\right) / 128 D_{1}^{9 / 2} .
\end{gathered}
$$

We have also investigated the representation

$$
\epsilon_{0}^{-1 / 2}(x)=\frac{F_{1}+F_{2} x+F_{3} x^{2}+b x^{3}}{1-\left(F_{4} / F_{1}\right) x^{3} \ln x+a x^{3}}
$$


for which the two double-pole conditions (13) and (14) imply that

$$
b=-x_{r c c p}^{-3}\left[F_{1}+F_{2} x_{r c c p}+F_{3} x_{r c c p}^{2}\right]
$$

and

$$
F_{2}+2 F_{3} x_{r c c p}+3 b x_{r c c p}^{2}=-\left(\frac{3}{5 x_{r c c p}}\right)^{\frac{1}{2}}\left(\frac{3}{\pi \nu}\right)^{\frac{1}{3}}\left[1-\frac{F_{4}}{F_{1}} x_{r c c p} \ln x_{r c c p}+a x_{r c c p}^{3}\right] .
$$

The values of $a$ and $b$ so determined are -0.0924883 and 0.171942 , respectively. This representation is unsatisfactory because it has what applied mathematicians call a "defect." Unfortunately it is in the physical region $0<x<x_{\text {rccp }}$. The problem is not uncommon and stems from a pole and a zero lying very close to each other.

\section{Conclusions}

Based on known terms of field-theoretic perturbative low-density expansions we have constructed closed-form analytical expressions as functions of particle density using Padé and other approximants for the energy per particle of the fluid branches of both many-boson and many-fermion quantum hard-sphere systems. Improvements with respect to previous work (notably but not exclusively that of Ref. 24]) have been achieved by assuming i) that the classical random closest close-packing hard-sphere densities are the ultimate fluid densities at which the energy diverges with a second-order pole and ii) proposing and imposing a value for the residue at the pole that is the same for either bosons or fermions as closest close-packing is approached and the hard spheres become distinguisable. Implementing these two conditions and taking advantage of recent diffusion Monte Carlo simulation data has allowed us to incorporate an additional term in the low-density expansion beyond that employed in Ref. [24]. The resulting determination of the best approximants has produced decidedly improved results for bosons as well as for two-component fermions, but not for four-component fermions.

\section{ACKNOWLEDGMENTS}

MAS thanks Washington University for hospitality during a sabbatical year and support as an Edwin T. Jaynes Visiting Professor. MdeLl thanks the NSF (USA) for partial support through grant INT-0336343 made to the Consortium of the Americas for Interdisciplinary Science, University of New Mexico, Albuquerque, NM, USA. We also acknowledge research support from UNAM-DGAPA-PAPIIT (Mexico) grants IN106401 and IN108205, from CONACyT (Mexico) grants 27828-E and 41302-F, and from NSF (USA) grant PHY-0140316. This work was also supported in part by the U.S. Department of Energy at the Los Alamos National Laboratory under Contract DE-AC 52-06NA25396. 


\section{References}

[1] F. London, Superfluids Vol. II (Dover, New York, 1964) p. 30.

[2] C.A. Rogers, Packing and Covering (Cambridge University Press, Cambridge, UK, 1964).

[3] N.J.A. Sloan, Sci. Am. 250, (Jan. 1984) p. 116.

[4] T.C. Hales (2003) A Proof of the Kepler Conjecture. See PDF in http://en.wikipedia.org/wiki/Kepler_conjecture\#References

[5] J.G. Kirkwood, E.K. Maun, and B.J. Adler, J. Chem. Phys. 18, 1040 (1950).

[6] C.N. Yang, Physica 26, S49 (1960).

[7] J.B. Hubbard, L. Masinter, and Z.W. Salsburg, J. Chem. Phys. 51, 4363 (1969).

[8] W. Lenz, Z. Phys. 56, 778 (1956).

[9] E.H. Lieb and J. Yngvason, Phys. Rev. Lett. 80, 2504 (1998).

[10] M.A. Solís, M. de Llano, and R. Guardiola, Phys. Rev. B 49, 13201 (1994).

[11] R.K. Cole, Jr., Phys. Rev. 155, 114 (1967).

[12] M.H. Kalos, D. Levesque, and L. Verlet, Phys. Rev. A 9, 2178 (1974).

[13] S.Z. Ren and M. de Llano, Eur. J. Phys. 10, 96 (1989).

[14] A.L. Fetter and J.D. Walecka, Quantum Theory of Many-Particle Systems (McGraw-Hill, New York, 1971).

[15] T.D. Lee, K. Huang, and C. N. Yang, Phys. Rev. 106, 1135 (1957).

[16] T. T. Wu, Phys. Rev. 115, 1390 (1959).

[17] G.A. Baker, Jr., Rev. Mod. Phys. 43, 479 (1971).

[18] G.A. Baker, Jr., L.P. Benofy, M. Fortes, M. de Llano, S.M. Peltier, and A. Plastino, Phys. Rev. A 26, 3575 (1982).

[19] G.A. Baker, Jr., M. de Llano, and J. Pineda, Phys. Rev. B 24, 6304 (1981).

[20] G.A. Baker, Jr. and P.R. Graves-Morris, in Padé Approximants, 2nd ed., edited by G.-C. Rota (Cambridge University Press, New York, 1996).

[21] V.C. Aguilera-Navarro, R. Guardiola, C. Keller, M. de Llano, M. Popovic, and M. Fortes, Phys. Rev. A 35, 3901 (1987).

[22] M.A. Solís, V.C. Aguilera-Navarro, M. de Llano, and R. Guardiola, Phys. Rev. Lett. 59, 2322 (1987).

[23] G.A. Baker, Jr., G. Gutiérrez, and M. de Llano, Ann. Phys. (N.Y.) 153, 283 (1984).

[24] C. Keller, S.Z. Ren, M. de Llano, M.A. Solís, and G.A. Baker, Jr., Ann. Phys. (N.Y.) 251, 64 (1996).

[25] S. Torquato, T.M. Truskett, and P. G. Debenedetti, Phys. Rev. Lett. 84, 2064 (2000).

[26] G.D. Scott and D.M. Kilgour, Br. J. Appl. Phys. 2, 863 (1969).

[27] H.M. Jaeger and S.R. Nagel, Science 255, 1524 (1992).

[28] V.C. Aguilera-Navarro, S. Ho, and M. de Llano, Phys. Rev. A 36, 5742 (1987).

[29] S. Giorgini, J. Boronat, and J. Casulleras, Phys. Rev. A 60, 5129 (1999). 
[30] Z. Hu, M. de Llano, E. Buendía, and R. Guardiola, Phys. Rev. B 43, 12827 (1991).

[31] M.A. Solís, R. Guardiola, and M. de Llano, in Cond. Matt. Theories, Vol. 8, (Plenum Press, 1992) p. 215.

[32] R.A. Aziz et al., J. Chem. Phys. 70, 4330 (1979).

[33] J.A. Barker and D. Henderson, J. Chem. Phys. 47, 2856 (1967).

[34] R.M. Panoff and J. Carlson, Phys. Rev. Lett. 62, 1130 (1989).

[35] G.A. Baker, Jr. and L.P. Benofy (priv. comm.).

[36] A. Fabrocini, S. Fantoni, A. Polls, and S. Rosati, N. Cim. A 56, 33 (1980)

[37] G.A. Baker, Jr., L.P. Benofy, M. Fortes, and M. de Llano, Phys. Rev. C 34, 678 (1986).

[38] L.P. Benofy, J.L. Gammel, R.M. Delaney, and G.A. Baker, Jr., Phys. Rev. C 48, 2685 (1993). 